\title{
Le nu au service de la santé
}

Gilbert Andrieu

\section{(2) OpenEdition}

Journals

Édition électronique

URL : http://journals.openedition.org/trema/2108

DOI : 10.4000/trema.2108

ISSN : 2107-0997

\section{Éditeur}

Faculté d'Éducation de l'université de Montpellier

\section{Édition imprimée}

Date de publication : 1 décembre 1995

Pagination : 3-14

ISSN : 1167-315X

\section{Référence électronique}

Gilbert Andrieu, « Le nu au service de la santé », Tréma [En ligne], 8 | 1995, mis en ligne le 20 septembre 2013, consulté le 01 mai 2019. URL : http://journals.openedition.org/trema/2108 ; DOI : 10.4000/trema.2108

Ce document a été généré automatiquement le 1 mai 2019.

Trema 


\title{
Le nu au service de la santé
}

\author{
Gilbert Andrieu
}

1 À la fin du XIX siècle et au début du $\mathrm{XX}^{\mathrm{e}}$ siècle, pour un ensemble de raisons que nous évoquerons plus tard, le nu semble s'imposer et soutenir un effort constant en faveur de la santé. Cette association qui peut paraitre étrange aujourd'hui mérite analyse, et c'est ce que nous allons essayer de faire sans nous laisser influencer par des interprétations hâtives, dues essentiellement à une sensibilité de fin de siècle ${ }^{1}$. Le nu, ne sous-estimons pas sa force suggestive, nous interpelle affectivement bien avant que nos efforts d'objectivation ne soient éveillés. Or, ce que nous souhaitons évaluer essentiellement n'est pas la nature du beau qui se montre dans de nombreux manuels d'éducation ou de culture physique, mais le pourquoi de son utilisation. Reconnaissons au passage qu'une telle utilisation apparait seulement à la fin du siècle et se vulgarise, pour les deux sexes, au début $\mathrm{du} \mathrm{XX}^{\mathrm{e}}$ siècle, à une époque où le nu reste confidentiel. Soulignons également que ce nu se montre dans des publications qui peuvent apparaître comme des publications adressées au grand public et recherchant une certaine publicité. Il nous faudrait ici analyser le sens de l'image dans cette société qui s'ouvre à la fois aux rigueurs d'un positivisme envahissant autant qu'aux effets morbides d'une économie fondée sur l'industrie ${ }^{2}$.

2 En dehors du nu pervers qui garde sa force de persuasion, le « nu pédagogique » peut être considéré comme une nouveauté significative ou représentative d'un contexte particulier. Parler du nu est une chose, le montrer en est une autre et nous devons éviter de nous méprendre sur les raisons qui poussent certains à l'utiliser. Car c'est bien ainsi qu'il faut l'entendre, et c'est d'un «nu utile » que nous parlerons ici exclusivement. Permis par l'art de la photographie, le «nu pédagogique» doit être en effet considéré comme l'illustration d'un idéal possible, un idéal qui sera comparé, avec plus ou moins de bonheur, à celui de la Grèce antique perceptible à travers la statuaire élevée au rang de valeur symbolique.

3 Pour comprendre l'usage de la photographie du nu masculin ou féminin dans les manuels de culture physique ou d'éducation physique, il faudrait nous attarder non seulement sur les discours des esthètes mais aussi et surtout sur ceux des hygiénistes ou des 
physiologistes auxquels il faudrait associer ceux des philosophes ou des sociologues comme Bergson ou Durkheim ${ }^{3}$. Mais c'est principalement dans l'observation des photographies que nous souhaitons trouver l'essentiel d'une explication qui, pour nous, semble se résumer en quelques mots : la santé est belle à regarder, ou encore : pas de beauté en dehors de la santé. Reste, bien entendu, à définir la santé à cette époque et à maîtriser les dessous d'une définition qui peut sembler provenir d'un choix dont nous ne percevons pas toujours l'objectivité.

4 En devenant symbole de santé, la beauté nue devient également symbole de liberté, de vérité, de pureté et s'oppose à toutes les pressions culturelles à un moment où le besoin de prendre quelques distances vis-à-vis du progrès s'impose. Le corps, libéré de ses entraves culturelles ou seulement vestimentaires, représente, au-delà d'un souci d'objectivation, une volonté d'émancipation, de renaissance et, secondairement, de régénération. L'homme nu c'est aussi l'homme naturel, l'homme non encore déformé et trahi par le progrès matériel, c'est l'homme pur non encore entraîné vers toutes les turpitudes d'une socialisation trop rapide et d'une urbanisation incontrôlée, c'est enfin l'homme du passé, celui dont la mémoire n'a retenu que le meilleur ${ }^{4}$.

5 Le nu devient miroir d'une vérité cachée et nous pourrions dire sans trop d'exagération, que les manuels utilisant le nu intégral, et non des dessins, ont un caractère subversif. A côté du sport qui se développe, à côté des gymnastiques médicales ignorées du grand public, à côté des gymnastiques militaires, à côté des gymnastiques revanchardes, les pratiques exposant la nudité comme une enseigne choquent, dérangent, questionnent et subjuguent par leur message simple, direct, compréhensible par les moins instruits.

6 C'est cette impression particulière que nous aimerions retrouver ici, l'explication pouvant être considérée comme secondaire.

\section{La nudité au service du peuple}

7 Notre première impression découle de la nature même des ouvrages qui utilisent le nu à des fins pédagogiques. Alors que les manuels ordinaires d'éducation physique ou de gymnastique analytique font état de corps habillés, les manuels de culture physique abordent le nu comme un étendard qui pourrait bien signifier la revanche des humbles. 
Un pied accroché à la barre, mains aux hanches : flexion latérale du tronc, du côté opposé à la barre. Exercices de flexion latérale. Barre à hauteur des hanches.

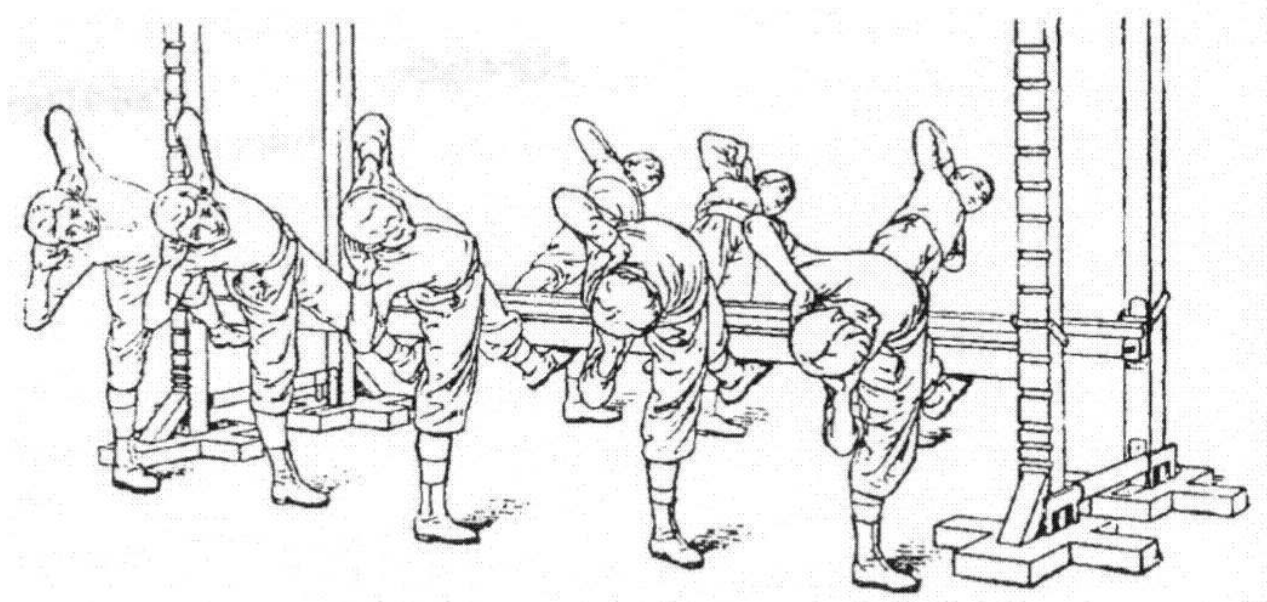

Ministère de I'Instruction Publique, Manuel d'Exercices Physiques et de Jeux Scolaires, Paris, Hachette, 1 réd., 1908.

Lorsque Desbonnet choisit de se faire le chantre de la culture physique et de fonder celleci sur le culte de la force athlétique, c'est pour un peuple opprimé par le travail et soumis aux effets de l'alcool et du tabac qu'il travaille et non pour la bourgeoisie au pouvoir, celle qui vient de décréter la gymnastique obligatoire à l'école pour des raisons essentiellement militaires. Cette opposition, ordinairement négligée, est à l'origine d'un certain nombre de différences qui ne sont pas l'effet du hasard. Nous pourrions voir le même type d'opposition, à la même époque, entre le développement du sport et celui des jeux scolaires de P. Grousset. À travers les pratiques corporelles et les représentations du corps, deux classes sociales s'affrontent et deux idéaux, l'un aristocratique et bourgeois, l'autre populaire, sont opposés et défendus par quelques initiés qui se comportent comme des croisés voulant refaire le monde.

Laissons de côté pour le moment les propositions bourgeoises, les plus savantes, celles qui font référence aux valeurs à la mode et tout particulièrement à la physiologie positive, et intéressons-nous principalement aux autres, celles qui s'adressent à ceux qui ne savent pas toujours lire et que l'image peut encourager ${ }^{5}$.

10 Remarquons toutefois que dans les ouvrages de la première catégorie, les images sont là pour bien montrer ce qu'il faut faire, car la gymnastique officielle est affaire de volonté et de discipline, d'ordre et de morale, et que dans les ouvrages de la seconde catégorie, les images sont là pour suggérer un idéal, faire rêver tout en laissant entendre que le rêve peut devenir réalité. Les uns fonctionnent à partir de la raison, les autres de l'imagination et des sentiments. Le nu se trouve du côté des émotions et non du côté d'une maitrise de soi qui empoisonnera tout le $\mathrm{XX}^{\mathrm{e}}$ siècle.

11 C'est probablement pour cela que les premiers manuels sont considérés comme sérieux tandis que les seconds sont qualifiés de prospectus commerciaux, ou de boniments pour lecteurs peu cultivés. Remarquons que nous avons tendance à comparer les uns et les autres à partir des normes scientifiques du moment sans prendre en considération le public auquel chacun s'adresse. L'opposition est alors faussée par des jugements qui n'ont pas de raison d'exister. Desbonnet et Tissié ne seront même pas comparés et pourtant ! Ils ont à l'origine de leur méthode la même influence, c'est-à-dire la gymnastique suédoise, 
mais leurs objectifs diffèrent et les outils qu'ils proposent pour régénérer la race, et la France en particulier, dépendent surtout des clientèles auxquelles ils s'adressent. Tissié, médecin, vise la jeunesse des écoles, Desbonnet, fils d'entrepreneur, vise la jeunesse au travail qui perd son temps libre dans les mes ou dans les cafés du Nord de la France. Nous pourrions opposer de la même façon P. de Coubertin, G. de Saint

Clair ou le père Didon et $P$. Grousset, plus tard G. Hébert et E. Desbonnet et, pourquoi pas, dans les années $1880, \mathrm{G}$. Rouhet et $\mathrm{F}$. Lagrange pourtant médecins de campagne tous les $\operatorname{deux}^{6} \ldots$

Ce que nous traitons ordinairement, en histoire de l'éducation physique, c'est le discours savant, le discours officiel ou académique, celui qui s'adresse aux enfants de l'élite et néglige les autres. Il suffit de relire les textes écrits par Coubertin ou Didon, ceux des médecins de l'Académie de Médecine prenant pour cible le surmenage scolaire, ceux de nos ministres accompagnant la création des Bataillons Scolaires, etc. ... pour voir qu'ils se soucient peu des enfants du peuple autrement que sous la forme de capital humain. Le bonheur vient après le devoir!

Pendant longtemps encore la force utile sera l'objectif principal de ces réformateurs qui oublient de faire du social. C'est un homme artificiel, une machine compliquée, mais une machine tout de même, qui reste l'objectif premier des concepteurs de méthode, des défenseurs du mouvement et nous ne nous étonnerons pas de voir le beau rationnel prendre le pas sur le beau viscéral, la beauté se soumettre aux lois de la physiologie et perdre sa charge émotionnelle. Le merveilleux doit s'effacer devant l'analyse scientifique et c'est ainsi que Demeny se laissera entrainer, par la chronophotographie, vers un mouvement tout aussi artificiel que les autres. Et pourtant, musicien, il écrira des pages très justes sur l'art de jouer du violon... En restant positiviste il perdra la possibilité d'enfanter une gymnastique véritablement humaine, une gymnastique possédant une âme.

15 Mais, positiviste, comme il était de bon ton de l'être au début de la Troisième République, Demeny ne pouvait pas faire passer la mélodie avant le rythme, l'harmonie des formes et la grâce des mouvements avant l'harmonie des fonctions et l'économie des forces. Pour lui comme pour tous les esthètes de son temps, l'idéal réside dans l'absence des stigmates de l'effort. L'essentiel est de paraître, plus que d'être, et nous dirons que les gymnastiques analytiques, sur le plan esthétique, sont autant le reflet d'une distinction acquise que d'une hygiène de vie. Les privilégiés jouent avec leur corps, entre eux bien entendu, et se moquent de l'utilité de leurs actes. Leur beauté reste une beauté de convention. Les autres n'ont pas le choix. Ils doivent se développer pour servir les privilégiés, plus que la France, autrement dit le régime bourgeois mis en place depuis 1875. L'école du peuple sera obligatoire... non pour le bonheur du peuple mais pour la sauvegarde d'une République laïque, protestante, positiviste et capitaliste.

La vie d'E. Desbonnet nous éclaire sur des choix totalement opposés. Toute sa fortune et celle de sa mère seront nécessaires pour faire naître une prise de conscience loin des Académies et des Ministères. Comme Triat, son maître spirituel du Second Empire, mais avec moins de facilité, les temps changent et les modes avec, Desbonnet jouera la corde sensible, l'exemple, la vérité dénudée, l'exploit qui laisse rêveur. Triat faisait travailler torse nu, Desbonnet fera travailler preque entièrement nu car il sait que l'individu est sensible à la force qui se montre bien plus qu'à la bonne marche des grandes fonctions. Pour la jeunesse populaire de la région de Lille, il donnera des leçons gratuites, il donnera le goût de la performance, le goût de la force individualisée et des formes athlétiques qui 
l'accompagnent. Il donnera le goût du plein air, de l'eau, du soleil, de l'action... Reprenant aux saltimbanques l'essentiel de leur préparation et de leurs tours, il fera naître le goût des gros poids et donnera naissance à l'haltérophilie pour donner plus de sérieux aux performances extrêmes.

Tandis que certains feront de l'escrime ou de l'équitation, d'autres des sports athlétiques, les fils du peuple apprendront à combattre les effets de l'alcoolisme par des pratiques roturières pour la simple raison qu'elles ne coûtent rien et qu'elles sont faciles à comprendre. Au peuple la force brutale, la force qui sent la sueur et la fonte, mais la force qui peut se lire sur des corps nus et possède depuis longtemps ses vedettes : les rois de la force ou de la lutte ${ }^{7}$. Cette distribution des pratiques et des pouvoirs ne doit pas être oubliée au moment où nous cherchons à comprendre les liens qui sont alors tissés entre l'image du nu et l'image de la santé.

\section{Les dessous de la santé}

18 L'image de la santé n'est pas la même pour tout le monde et l'image du nu qui l'accompagne, à la fin du XIXe siècle et au début du XXe siècle, non plus. Nous dirons pour commencer que la santé n'a pas le même sens pour les pratiquants tels que nous les avons classés dans le chapitre précédant. Disons que chaque image de la santé se perçoit essentiellement dans les discours qui s'efforcent d'argumenter la nécessité d'une pratique plus assidue, mieux adaptée aux besoins de l'homme moderne. Or, cela n'a pas changé depuis, les besoins sont décidés par ceux qui orientent le «plus de pratique », bien plus qu'ils ne sont établis à partir d'une observation de la réalité. L'homme moderne, tout comme l'athlète parfait ou complet, tout comme l'athlète antique, est un mythe, un objet manipulé qui ne reflète pas les différences, encore moins les attentes des futurs clients ${ }^{8}$.

L'image du nu nous enseigne à sa façon comment l'utilisateur doit envisager l'acquisition de la santé ou sa restauration. À côté du nu académique qui accompagne les manuels à usage médical, c'est le cas de l'hydrothérapie par exemple dès le Second Empire, autrement dit d'un nu sans pulsions, d'un nu sans âme, à côté d'un nu grivois qui abonde dans les revues qui tentent de prendre à revers la pudibonderie bourgeoise, à côté du nu romantique qui prend place dans les revues traitant de la nature, tout particulièrement de la montagne, il y a le nu, en chair plus qu'en os, qui ose affronter le nu antique et sert de symbole pour rendre plus crédible l'idée d'une beauté synonyme de santé.

A côté du nu presque intégral, il y a les gravures qui représentant le corps figé dans une posture ou une phase particulière du mouvement. Ces différentes illustrations donnent un sens au pourquoi de l'acte régénérateur et dévoilent les divergences au-delà des caractéristiques communes. Pourtant, toutes les représentations du nu sont mises au service d'une notion qui semble s'imposer également pour tous: la santé. Nous disons notions car, plus qu'un fait observable, la santé se présente d'abord comme une démonstration, une vérité scientifique, une qualité utile, secondairement comme un état particulier qui peut varier d'un utilisateur à l'autre.

Or, sur le plan esthétique, l'image du nu intervient pour fixer les contours objectifs de cette notion, elle lui donne une forme et cette forme revêt alors une fonction pédagogique certaine, et cependant changeante. Deux modèles académiques sont recrutés par les hygiénistes comme par les promoteurs de gymnastiques: l'Hercule Farnèse de Lysippe et le Gladiateur Combattant d'Agasias, le premier servant de contre preuve au 
second. L'Hercule gonflé de muscles au repos s'oppose au Gladiateur moins volumineux mais plus actif. L'athlète lourd, tout en puissance, subit la défaite que lui impose une société en voie d'industrialisation, tandis que l'athlète léger, en apparence plus déterminé puisqu'il défend sa propre existence, bénéficie d'un éclairage physiologique qui pourrait nous surprendre.

Certes le gladiateur semble plus conforme à l'idée que l'on se fait d'un combattant moderne et semble plus apte à se déplacer rapidement, quant à dire qu'il respire mieux, par exemple, la statuaire antique ne le dit pas. Que faudrait-il penser de Milon de Crotone courant avec un bœuf sur ses épaules? Aussi nous ne nous laisserons pas séduire par de subtils glissements de la fonction à la forme.

Ce qui apparaît surtout, chez ceux qui vantent l'athlète léger, c'est la volonté d'imposer au lecteur un style de vie en rapport avec un type de corps! L'image du nu, parce qu'elle est antique, guide l'esprit plus que les sens vers un idéal économique et moral propre à la Troisième République. Constatons, sans plus attendre, que les deux promoteurs de méthodes nouvelles qui utilisent véritablement le nu pour vendre leur produit, associent étroitement le nu antique et des corps modernes dénudés, souvent figés dans des attitudes comparables. Desbonnet et Hébert sont aussi des guides qui ne sacrifient pas sur l'autel de la science l'émotion que suggère un corps semblable à celui qui le regarde, un corps capable d'engendrer le désir. Car il y a là une différence importante: les uns suggèrent le travail, l'effort, l'attention, la volonté, le dépassement permanent dans une atmosphère toute ascétique lorsqu'elle n'est pas franchement militaire; les autres suggèrent l'effort, l'attention, la persévérance, la rigueur dans une ambiance de plaisir immédiat et de bonheur futur. Les corps sont des objets surveillés, modelés par d'autres dans le premier cas; les corps sont sujet dans le second cas, ils sont libres de jouir en toute innocence. À vrai dire, les corps dénudés de G. Hébert sentent encore le renfermé, le musée, la norme, ils ne sont pas libérés et c'est probablement là que l'auteur de la méthode naturelle se trahit. Ceux d'E. Desbonnet, en particulier les femmes, sont plus détendus, ils s'amusent, prennent le bain ou simplement le bain d'air, ils invitent à l'amour plus qu'à la guerre. Mais ne nous laissons pas séduire trop vite, car ce beau enjôleur vise un but : celui de la meilleure procréation possible.

Faut-il ajouter que les poses masculines sentent l'argumentation plus que l'émotion esthétique. La faiblesse des comparaisons, entre l'Antiquité et la modernité, est probablement la plus grande source de critiques. Sans poser, les athlètes modernes auraient probablement plus de charme et c'est ce qui se passe pour certains d'entre eux. Disons que, libérés du poids de l'Antiquité, les culturistes des deux sexes sont plus proches de nous.

Où est la santé dans chaque cas et quelle santé ? Pour les plus conservateurs, la santé d'un corps figé semble indiquer la surveillance de la colonne vertébrale, son redressement et le contrôle de la volonté sur tous les gestes exécutés, le maitre mot pourrait être la discipline, à moins que ce ne soit l'autodiscipline. Pour les corps habillés, comment ne pas souligner la distinction qu'apporte l'uniforme, qu'il soit celui des militaires, des gymnastes ou bien des premiers sportifs, après l'intervention de G. de Saint Clair !?

Pour les moins conservateurs, les plus révolutionnaires peut-être mieux, les corps dénudés nous mettent en présence d'une santé belle à regarder, une santé qui accorde au relief musculaire une place importante. La photographie fige le temps et stoppe le mouvement. En regardant une photographie de corps nu, ce n'est pas le mouvement qui se montre mais l'harmonie des formes, l'impression d'aisance et de force, l'attitude avec 
ses divers degrés de relâchement, les traits du visage, le grain de la peau... Tandis que le marbre refroidit la chair la photographie l'enflamme, notre imagination galope et si, naturellement, la nature nous guide vers la beauté qui respire, convenons qu'il faut être esthète pour aimer sensuellement la beauté éternelle des sculptures antiques.

Santé mécanique et fonctionnelle d'une part, santé érotique d'autre part, la divergence ne cessera de grandir entre les tenants d'une santé au service de la société et une santé au service du plaisir. La santé qui s'habille ne serait-elle pas illusoire?

\section{Le droit de s'aimer}

Ce que les photographies d'athlètes nus ou de culturistes nues suggèrent encore, c'est, audelà du plaisir, le droit de s'aimer". Ce retour sur soi, qui ne cessera d'être critiqué et excitera les psychanalystes tout au long $\mathrm{du} \mathrm{XX}^{\mathrm{e}}$ siècle, est probablement une réaction à l'enfermement des individus dans des normes physiques et morales toujours plus contraignantes. En remplaçant le goupillon par le sabre, la bourgeoisie bien pensante du $\mathrm{XIX}^{\mathrm{e}}$ siècle a certainement joué un rôle dans l'émergence d'un besoin d'air, à tous les sens du terme. Agir nu, se laisser photographier nu, accepter d'afficher aux yeux de tous un corps régénéré... c'est aussi prendre le parti d'une vérité cachée qui cherche à se faire entendre.

29 Le sportif trouve son plaisir dans la victoire, le culturiste le trouve dans la métamorphose de son être. Le plaisir est volé au sportif par le public, tout aussi friand de défaite, le plaisir est donné par le culturiste à tous ceux qui ne sont pas encore totalement dénaturés. Pour que le culturiste fasse don de sa beauté, il faut d'abord qu'il apprenne à s'aimer car cela s'apprend. Il n'y a pas de métamorphose sans effort, et cet effort n'est accepté que parce qu'il s'accompagne d'un plaisir chaque jour plus grand. Cet amour de soi, loin d'être une régression, est un véritable excitant et Desbonnet l'a bien compris. En faisant travailler ses élèves nus devant des glaces, il a joué la carte du spectacle ${ }^{10}$. Chaque élève mis face à lui-même sait immédiatement qu'il travaille pour lui et il est le premier à rencontrer l'émotion esthétique qui le guidera jusqu'au terme d'une création.

Les photographies d'hommes et de femmes nus que nous rencontrons dans les livres d'Hébert ou de Desbonnet, sont d'abord des photographies d'hommes et de femmes qui s'aiment assez pour accepter le viol d'un appareil photographique. C'est parce qu'ils n'ont plus le besoin spontané de se cacher qu'ils acceptent de paraître nus devant d'autres, d'être aimés par d'autres. Ils se donnent parce qu'ils ont acquis cette paix intérieure qui leur permet de vivre la relation dans une ambiance de vérité, de pureté morale. Un corps revenu à l'état de nature, celui de l'athlète et non du dégénéré, dans une nature non dénaturée, devient le symbole du bonheur et de la liberté, combat que les nudistes devront mener entre les deux guerres mondiales avant de se parquer pour vivre autrement. Bien entendu, l'exploitation du nu et de la statuaire antique simultanément conduira à des excès risibles, à des fautes de goût, à un défaut de vérité qui nuit au sujet photographié tout autant qu'il déplaît à l'observateur.

31 Aussi pourrions-nous dire ici que les athlètes d'Hébert sont peut-être moins dérangeants que les faux hercules de Desbonnet. En revanche, les femmes de Desbonnet sont moins dérangeantes que celles d'Hébert, tout simplement parce qu'elles restent conformes aux canons du plaisir. 
En apprenant à aimer son corps, la femme chez Desbonnet apprendra à choisir un bon mari, chez Hébert, la Grande Guerre les sépare, la femme forte et belle n'apprend-elle pas à se passer de mari?

Fente latérale fléchie et fente arrière fléchie. Exercices physiques pour élèves de 6 à 13 ans.
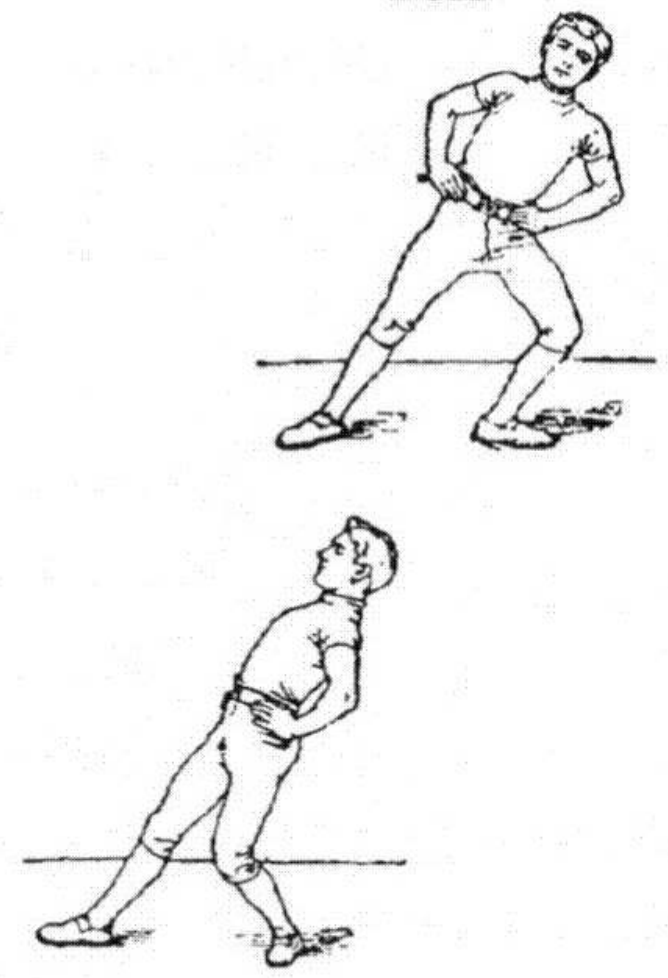

Ministère de I'Instruction Publique, Manuel d'Exercices Physiques et de Jeux Scolaires, Paris, Hachette, 1 réed., 1908.

Après tout, il est permis de rêver, d'imaginer une société faite d'hommes et de femmes nus, procréant des enfants nus dans une nature respectée et dans le plus profond désir de vivre heureux. Lorsque nous regardons les photographies de nu proposées par ces deux auteurs, peut-être pas toutes, nous sentons bien que derrière l'émotion ressentie, loin de toute influence culturelle figée, elles nous invitent à partager le secret d'une autre forme de bonheur, un bonheur sans violence, un bonheur fondé sur la communion des âmes et des corps. Ces photographies nous montrent qu'il n'est pas interdit d'aimer, bien au contraire, et nous dirons que ceux qui rient jaune en les regardant sont trop malheureux pour comprendre ce message.

Tout compte fait, le nu révèle ici la santé mentale bien plus que la santé physique. Les formes ne sont que le reflet, l'image d'un équilibre acquis par le travail sans contrainte et n'échauffent notre imagination que parce que nous avons perdu la possibilité de comprendre. Si nous sommes choqués, c'est bien souvent parce que nous ne voulons pas avouer notre misère et que nous avons peur de remettre en question les valeurs auxquelles nous sommes attachés.

Il fallait beaucoup de courage à Desbonnet, comme à Hébert, pour illustrer leurs manuels avec des corps nus véritables et pas seulement des dessins ou des reproductions d'œuvres d'art. Ces photographies agressent encore aujourd'hui, du moins ceux qui ont refusé 
d'être ce qu'ils sont avant de penser : des êtres qui réagissent instinctivement et sont sensibles à la beauté quand elle se montre ${ }^{11}$

\section{Conclusion}

Ces quelques lignes, je les offre à tous ceux qui ne sont pas tombés dans les pièges des mots et qui savent tendre la main du cœur quand la beauté se présente. Je les offre également à tous ceux qui se croient libérés parce qu'ils évaluent des connaissances sans âme. À ceux qui ne supportent pas de voir un corps nu, qui n'ont pas de passion et se cachent derrière des techniques, je voudrais dire qu'enseigner la vie c'est enseigner l'amour de soi et des autres, c'est enseigner le respect des autres à travers l'image que nous leur offrons quotidiennement. Desbonnet avait compris le premier, ce sens de la vie, et nous nous sommes empressés de le décrier pour nous doter d'une aura scientifique. Le sport, en devenant de plus en plus technique, nous permettait d'éloigner plaisir et désir spontané, bonheur d'être deux, joie simple et profonde d'être beau... Nous sommes devenus docteur, mais de quoi !

37 Je doute qu'il soit possible d'oser plus que Desbonnet ou Hébert, tout simplement parce qu'ils ont touché l'individu que nous sommes au bas-ventre et que toute construction humaine sérieuse ne peut ignorer cet étage fondamental de la personnalité. Avant de chercher à transcender la vie, il faut jouir et c'est peut-être ce que le $\mathrm{XX}^{\mathrm{e}}$ siècle a le mieux refoulé.

Notre santé, aujourd'hui comme toujours, dépend au premier chef de notre beauté physique. Je dirai qu'on ne peut réussir sa vie sans se plaire, l'école l'a trop oublié.

Le " Pont » abaissé et relevé. Le pont abaissé, ou appui renversé sur les pieds et la tête, avec ou sans l'aide des mains.

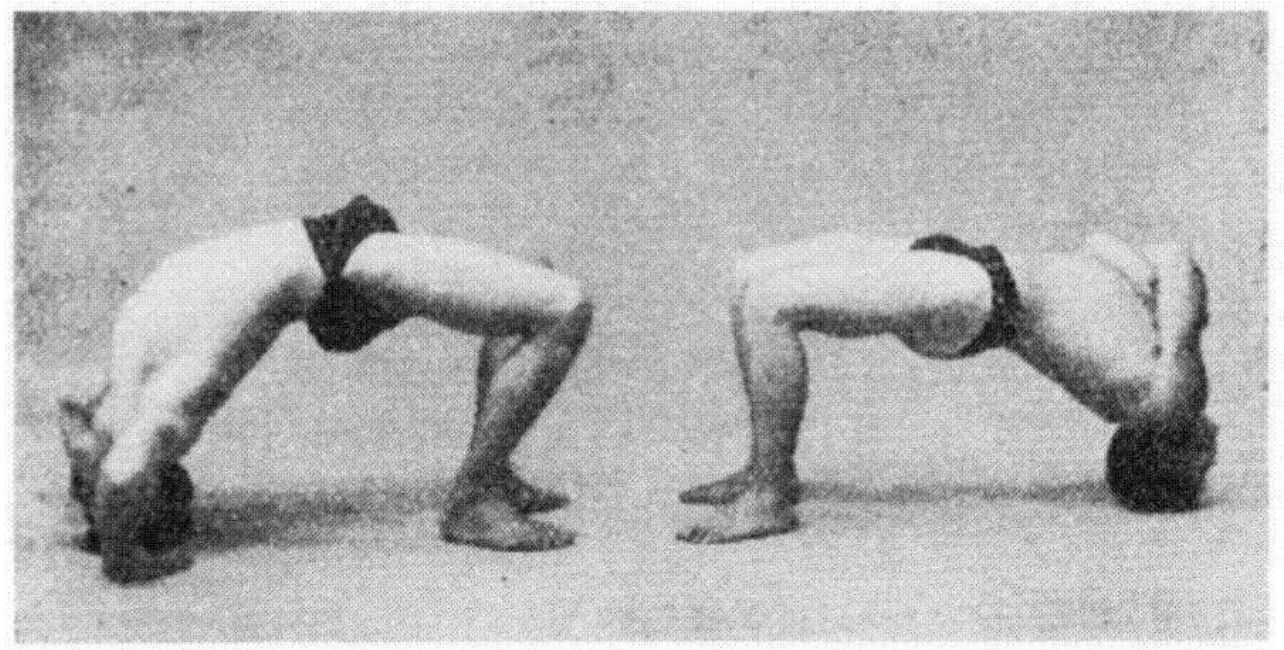

Georges Hébert, L'Education Physique Virile et Morale par la Méthode Naturelle, Tome I, Paris, Vuibert, 1936. 


\section{BIBLIOGRAPHIE}

ANDRIEU G. : L'homme et la force. Joinville-le-Pont, ACTIO, 1988.

ANDRIEU G. : Force et beauté. Histoire de l'esthétique en éducation physique aux XIX ${ }^{e}$ et XXe siècles.

Bordeaux, PUB, 1992.

CARTON Dr P. : Les lois de la vie saine. Paris, MALOINE, 1926 ( $2^{\mathrm{e}}$ éd.).

CARTON Dr P. : IM synthèse directrice et libératrice de la personne humaine, méthode hippocratique;

cartonienne Brevannes, chez l'auteur. 1925.

COUBERTIN P. de : Essais de psychologie sportive. Paris, Payot, 1913.

DEMENY G. : Les bases scientifiques de l'éducation physique. Paris, F. Alcan, 1902.

DEMENY G. : Physiologie des professions. Le violoniste. Paris, Maloine, 1903.

DEMENY G. : Mécanisme et éducation des mouvements. Paris, F. Alcan, 1903.

DEMENY G. : Éducation et harmonie des mouvements. Paris, Maloine. 1911.

DESBONNET E. : Comment on devient un athlète. Paris. B. Levrault. 1909.

DESBONNET E. : Les rois de la force. Paris, Lib. Athlétique. 1910.

DESBONNET E : Pour devenir belle et le rester Paris, B. Levrault, 1911.

DESBONNET E. et ROUHET G. : L'art de créer le pur-sang humain. Paris, B. Levrault, 1905.

DESCAMPS M. : Le nu et le vêtement. Paris, Éd. Universitaire, 1972.

DUFRENNE M. : Phénoménologie de l'expérience esthétique. Paris, PUF, 1953.

DURVILLE (frères) : Guide de la santé et de la beauté. Paris, Vivre-Santé, 1936.

GROUSSET P. : 1M santé par l'exercice et les agents physiques. Paris, O. Doin, 1889.

HEBERT G. : Le code de la force. Paris. Vuibert, 1911.

HEBERT G. : La culture virile et les devoirs physiques de l'officier combattant. Paris, Vuibert, 1913-

HEBERT G. : Muscle et beauté plastique féminine. Paris, Vuibert, 1911.

HEBERT G. : L'éducation physique virile et morale par la méthode naturelle. Tome I, Paris, Vuibert, 1936.

LAGRANGE Dr F. : Physiologie des exercices du corps. Paris, F. Alcan, 1888.

MAREY Dr E.J. : La machine animale. Paris, G. Baillière, 1873.

MAREY Dr E.J. : Le mouvement. Paris, Masson, 1894.

MONTEUUIS Dr A. : La médecine naturelle.... Bruxelles, H. Lamertin. 1911.

NADEL H. : Devons-nous vivre nu ? La nudité et la morale. Paris, Vivre, 1930.

NORLANDER C. : Martin Éd., Manuel de gymnastique rationnelle suédoise à l'usage des écoles primaires. Bruxelles, Manceaux. 1883.

RICHER Dr P. : Physiologie artistique de l'homme en mouvement. Paris, Doin, 1895.

RICHER Dr P. : Nouvelle anatomie artistique du corps humain. 6 Tomes. Paris, Plon, 1926 (Tome 5). 
ROUHET Dr G. : De l'entraînement complet et expérimental de l'homme... Paris, Lib. Associés, 1902.

ROUHET Dr G. : IM jungle des civilisés. Paris, B. Levrault, 1925.

SOURIAU P. : L'esthétique du mouvement. Paris. F. Alcan, 1889.

SOURIAU P. : La beauté rationnelle. Paris, F. Alcan, 1904.

STREHLY G. : L'acrobatie et les acrobates. Paris, Delagrave, 1903.

\section{NOTES}

1. Il est très difficile, dans ce type d'observation, de ne pas mélanger objectivité et subjectivité. Devant un corps nu, d'homme ou de femme, nous avons tendance à réagir affectivement d'abord et nous oublions que notre approche du beau est fortement influencée par la culture et. plus peut-être, par la mode. Il n'y a pas de beau intemporel, notre goût dicte inconsciemment nos réactions et notre goût n'est que la synthèse d'un grand nombre d'influences. Il suffirait de considérer certains apollons de la fin du XIX siècle avec ceux d'aujourd'hui ou ceux de l'Antiquité, pour analyser les fondements de nos préférences. Les sculptures de Mayol ou de Belmondo nous parlent, comme celles de Rodin, d'une époque oubliée, et les photographies des manuels de culture physique ou d'éducation physique ne sauraient atteindre l'expression d'une œuvre d'art. Soulignons qu'elles n'en ont pas la prétention. En revanche, les sujets qu'elles figent sont si près de nous qu'ils dérangent et nous font prendre position, sans nuances, si nous n'y prenons garde.

2. Sans aller très loin dans la réflexion, disons que la photographie peut être considérée comme un outil scientifique. La chronophotographie permettra l'analyse du mouvement au laboratoire de Marey... Ici, la confrontation d'une multitude de clichés peut être considérée comme une approche expérimentale du corps, rationnelle, guidée par des critères d'observation et conduisant à des conclusions pédagogiques.

3. Il va de soi que l'analyse du beau puisse être retrouvée ça et là dans une infinie variété d'ouvrages. Ce que nous voulons faire ici, c'est privilégier les photographies et non la beauté à travers l'art. En le faisant, nous avons l'intention de nous rapprocher des auteurs qui ont utilisé ce type d'illustrations très suggestives. Desbonnet et Hébert ne sont ni des scientifiques, ni des artistes, ni des esthètes, mais ce sont des observateurs et des éducateurs qui ont su dépasser la simple démonstration, l'exemple, pour enseigner à la fois un idéal et un acte volontaire.

4. Il suffirait de comparer cette nudité à l'absence de nudité qui accompagne les progrès de la baignade, pour évaluer le degré d'émancipation qui, indirectement, donne un sens à la photographie.

5. La différence est grande entre ces deux clientèles et cela n'est pas nouveau. Notre analyse des foires et des commerces du corps sous le Second Empire nous a montré que le peuple recherche l'émerveillement, qu'il aime admirer et craindre sans comprendre, alors que les classes cultivées gouvernent leurs actes à l'aide de choix réfléchis, argumentés, partagés, non plus sur des émotions mais sur celui des analyses. Les propos de Strehly sont significatifs lorsqu'il nous parle des saltimbanques ou du cirque.

6. L'année 1888 est riche en oppositions et pourrait à elle seule devenir objet de recherche. Les publications scientifiques, les réactions médicales, mais surtout les innovations en faveur du mouvement font apparaître des choix très différents, des choix qui trouvent leurs racines dans des enjeux politiques et économiques importants,

7. N'oublions pas que si l'analyse chronophotographique change la façon de regarder le corps en mouvement, elle ne touche qu'une minorité de personnes, tandis que les exploits des forains restent visibles pour une masse de badauds à la recherche d'un peu de rêve. Entre le peuple et 
cette force brutale, il n'y a que l'épaisseur du tour qui n'utilise pas toujours l'art de la tricherie. La comparaison est parfois facile entre l'acteur et le spectateur : il suffit de poser la chemise et d'essayer...

8. Le beau et sa justification hygiénique apparaissent alors comme des vérités incontournables, des vérités qui doivent être admises bien avant d'être observées. Qu'il s'agisse des esthètes dissertant sur la beauté plastique ou la grâce des mouvements, qu'il s'agisse des scientifiques affirmant que la beauté découle de la santé, qu'il s'agisse des promoteurs de méthodes..., le beau athlétique est décrit plus que montré, il dérive de choix qui ne sont pas toujours rationnels, il est un mythe adroitement manipulé par ceux qui le créent.

9. Une telle affirmation peut surprendre. Pourtant, ne sommes-nous pas habitués, dressés, depuis plus d'un siècle, à être des insatisfaits permanents, des infirmes dépendant d'analyses religieuses ou scientifiques morbide? Dans un monde où l'homme apprend sans cesse à s'autodévaloriser, comment pourrait-il accéder à une vision régénératrice qui vise l'ensemble des discours à la mode? Reconnaissons que la psychologie de l'attention ou de la volonté, la psychanalyse, la sociologie sont déjà l'essentiel d'une pression, plus lourde encore que la pression religieuse, et que, devant cette pression, le nu a du mal à se faire admettre.

Quant au retour sur soi, que la pédagogie du nu semble induire, il ne saurait être entendu facilement au moment où l'homme devient un produit de la société.

10. Le spectacle auquel assiste le culturiste devant sa glace, est ordinairement bien connu des danseurs. Desbonnet n'invente rien. Il utilise parfaitement l'objet en ce sens que la glace n'est pas un simple miroir qui renvoie l'image de l'acteur, elle renvoie également une image de soi qui sert de base à toutes les comparaisons et à toutes les transformations possibles. Elle devient enseignant au sens socratique du terme, c'est-à-dire qu'elle permet à l'individu de naître et de devenir lui-même.

11. Tout n'est pas beau par principe et chacun de nous ressent la beauté non seulement à partir de ce qu'il sait, mais aussi à partir de ce qu'il est. N'oublions jamais que Desbonnet et Hébert, pour ne citer qu'eux, n'ont pas voulu faire de l'art proprement dit. Les photographies qu'ils utilisent ne sont pas des œuvres d'art, leur nombre suffit à confirmer leur vocation essentiellement pédagogique. Il serait bon d'analyser pourquoi elles nous gênent parfois, en dehors de toute évaluation esthétique et il ne faudrait pas oublier qu'elles datent...

\section{RÉSUMÉS}

Le nu à la fin du XIXe siècle et au début du XXe siècle fait son apparition dans de nombreux ouvrages consacrés à l'éducation physique et à la culture physique. Son utilisation est essentiellement pédagogique et esthétique. Le nu devient acceptable car il est avant tout démonstratif de la santé, d'une santé presque naturelle ou du moins associée à la nature. Le corps nu témoigne d'un homme non déformé 'et trahi par le progrès matériel. L'analyse de l'iconographie d'ouvrages de vulgarisation de la culture physique ou de l'éducation physique tels que ceux de Desbonnet ou Hébert, permet de définir la fonction globale, idéologique, du nu au cours de la période étudiée.

The nude in the Tate 19th and early 2,0th century begins to appear for the first time in numerous works devoted to physical education and to physical exercises. Its use is essentially pedagogical and aesthetic. The nude gains acceptance as it is first and foremost an expression of good health, 
of an almost natural or at any rate natural-like, type of good health. The nude body attests to man's body as being neither misshapen nor betrayed by material progress. Analysis of the pictorial corpus of works of popularization in the fields of physical education and physical exercises makes it possible to define the ideological and overall function of the nude over the course of the period studied.

INDEX

Mots-clés : histoire, Images, nature, nu, santé

Keywords : Health, history, nude

\section{AUTEUR}

\section{GILBERT ANDRIEU}

Professeur en S.T.A.P.S., faculté des Sciences du Sport de Bordeaux 